1. PhD

Assistant Professor

Riphah International University,

Islamabad, Pakistan.

2. $\mathrm{PhD}$

Professor

University of Lahore, Lahore,

Pakistan.

3. PhD

Assistant Professor

Islamia University of Bahawalpur Bahawalpur, Pakistan.

4. $\mathrm{PhD}$

Assistant Professor

University of Lahore, Lahore,

Pakistan.

5. PhD Scholar

University of Lahore, Lahore,

Pakistan.

6. PGR internal medicine

Shalamar Hospital, Lahore, Pakistan.

7. PGR internal medicine Arif Memorial Teaching Hospital, Lahore, Pakistan.

8. M.Phil

University of Lahore.

Correspondence Address:

Dr. Madiha Sikander

University of Lahore, Lahore, Pakistan.

dr.madihakhan83@gmail.com

Article received on:

24/01/2018

Accepted for publication:

$15 / 08 / 2018$

Received after proof reading:

04/01/2019

\section{SAFETY FIRST;}

A REVIEW

Muhammad Sikander Ghayas Khan', Arif Malik², Gull-e-faran ${ }^{3}$, Madiha Fayyaz ${ }^{4}$, Madiha Sikander ${ }^{5}$, Qurrat-ul-Ain $^{6}$, Rabia Ghayas ${ }^{7}$, Malik Muhammad Qasim ${ }^{8}$

ABSTRACT... Introduction: Laboratory is a base of developing teaching and scientific research, also a place of training practical and innovation ability. A robust laboratory experience is a very important part of a high-quality course of instruction in every subject for students interested in research and education. Therefore, a laboratory's physical design and layout must support and optimize investigation and synthesis of ideas and materials. Objectives: To guarantee safe practices among specialists in research about labs. Study Design: Review Article. Study Period: June 2017 to December 2017. Setting: Riphah International University, Lahore. Material and Methods: This review article was written to enhance the awareness of biosafety among the students and the professionals. A literature review was done for this study through searching original articles, reviews, case studies and different websites. After analyzing the literature, important aspects of biosafety were highlighted and conclusion was drawn. Results and Conclusion: Regardless of a few instructive workshops by the Pakistan Biological Safety Association (PBSA), consistence with safe practices among researchers stays low. The hazard for research facility authorities working with exceedingly risky operators ought to be limited through preparing and adherence to stringent security conventions and standard working techniques.

Key words: $\quad$ Laboratory Safety, Laboratory Accidents, Bio Hazards, Bio Security, Bio Safety.

Article Citation: Khan MSG, Malik A, Gull-e-Faran, Fayyaz M, Sikander M, Qurrat-ul-Ain, Ghayas R, Qasim MM. Safety first; a review. Professional Med J 2019; 26(1):1-6. DOI: 10.29309/TPMJ/2019.26.01.2581

\section{INTRODUCTION}

The improvement of student's awareness of concepts in science and its uses, practical scientific abilities and considerate of how science and scientists effort in laboratory experiences have been considered key aspects of education in science for over 100 years. Facility requirements for the necessary level of safety and security combined with specific requirements relevant to the course to be conducted dictate the fundamental strategy of a specific lab, and the strategy procedure need to address both. ${ }^{1}$

The creation of a laboratory-based training environment that allows students to plan examinations, take part in logical thinking, control hardware, record information, and investigations comes about, and talk about their discoveries. The focus of this particular article is to address basic biosafety concerns.
Authorities attention should brought towards the very important and neglected area of higher education institutes all over the world especially in Pakistan, "Laboratory safety"; hopefully this effort will develop the awareness about the urgency to implement of laboratory safety regulations in institutional laboratories and decreases the morbidity and motility ratio which is though high but remains unreported especially in Pakistan. "Safety First" is an important message for every laboratory worker. Guarding your working environment can fill numerous needs other than guaranteeing the wellbeing and prosperity of your representatives and guests. A protected workplace can help spirit, increment work fulfillment and lessen lost days and specialists reparation because of mishaps at work. A number of injuries arise inside the laboratory due to lack of proper expertise regarding laboratory protection measures, detached mindset \& wrong 
implementation of secure laboratory Practices. In most cases these dangers fall usually into one of the classes: organic, chemical, physical, electrical/ high voltage equipment, mechanical, equipment with shifting elements, psychological. ${ }^{2}$

Few are the examples of laboratory accident across the world.

A senior Yale University science student has been executed on 14 April 2011 after her hair got captured in a machine in an oddity mishap at a grounds research facility. Michele Dufault, 22, passed away because of asphyxia by neck pressure while utilizing a quick turning machine in the student machine shop at Sterling Chemistry Laboratory in New Haven, Connecticut. ${ }^{3}$ On Jan. 16, Sheharbano (Sheri), a 23-year-old science research assistant, died from wounds maintained in a compound fire on Dec. 29, 2008, in a lab at the University of California, Los Angeles. ${ }^{4}$ On Jan. 7, 2010, Texas Tech University (TTU) graduate student Preston Brown was attempting to incorporate and portray a vivacious material (nickel hydrazine perchlorate) when a blast happened. Brown lost three digits on his left hand, seriously gashed his right hand, punctured his left eye, scratched his right eye and had shallow cuts to the parts of his body that were exposed. ${ }^{5}$ By Richard Wronski and John Keilman Tribune staff journalists October 12, 2001 a blaze fire consumed seven students in a science class when an examination went away. ${ }^{3}$

These reported incidents actually warns the other stakeholders who are responsible for establishing institutional laboratories without following due safety Protocols.

\section{Institutional Biosafety Committees}

There are many authorized organizations dealing with Laboratory safety measures implementation in the regarding institutes such as National biosafety center which further regulates The Pakistan Biosafety Rules, 2005, which concerns genetically modified organisms, built up the accompanying elements: The National Biosafety Committee whose obligations (identified with genetically modified organisms) The Technical
Advisory Committee that looks at applications and gives exhortation concerning deal with and the arrival of genetically modified organisms. The Pakistan Biological Safety Association is a nonbenefit, non-administrative expert association devoted to the arrangement of exhaustive learning identified with biosafety issues in Pakistan. These organizations conduct workshops, seminars and training programmes about biosafety for laboratory workers on regular basis.

The Pakistan Biosafety Rules 2005 require an association engaged with bio-innovation or genetic manipulation to assign a Biosafety Officer.

Pakistan as of now has three Biosafety associations including the Pakistan Biological Safety Association (PBSA), which is partnered with the National Core Group in Life Sciences (NCGLS) of the Higher Education Commission and the Organization of the Islamic Conference (OIC) Committee on Scientific and Technological Cooperation (COMSTECH) and was begun in 2008.

Occupational Safety and Health Act (OSHA), was established by the U.S. Congress in 1970. The objective of this government control was to give all representatives (clinical lab faculty included) with a sheltered workplace. OSHA is approved to lead nearby investigations to decide if a business is agreeing to the obligatory models. ${ }^{4}$

\section{Trainings and Seminars}

Moreover, various preparing exercises and courses on biosafety have occurred in Pakistan, and Pakistanis have taken an interest in comparable occasions abroad. For instance, in 2009, Aga Khan University in Karachi held two courses on Laboratory Biosafety and a National Training Seminar on Biosafety and Biosecurity Initiatives occurred in 2007 in Islamabad. Likewise, Pakistanis went to the Asia Conference on Laboratory Biosafety and Biosecurity (Bangkok, Thailand, 2007); the Biosafety and Biosecurity International Conference: Healthier and More Secure Communities in the Middle East and North Africa Region (Casablanca, Morocco, 2009); the Biosafety and Biosecurity Risk Assessment and 
Risk Mitigation Training Event for Pakistani Bioresearchers (Dubai, United Arab Emirates, 2010); and the ICLS-COMSTECH-PAS International Conference on Conduct of Responsible Science: Safety, Security and Ethics (Islamabad, Pakistan, 2010). ${ }^{5}$
Researches on Awareness of Biosafety In Pakistan there are various studies conducted about the awareness about biosafety but still there is wanting in this particular field to arouse the importance of biosafety awareness. Some of these studies mentioned below in the following table.

\begin{tabular}{|l|l|}
\hline \multicolumn{1}{|c|}{ Author's Name \& year } & Studies conducted on awareness about laboratory safety \\
\hline Sadia Nasim Ahmed, 2012 & Biosafety perspective of clinical laboratory workers: a profile of Pakistan. (9) \\
\hline Abdul Salam Shah, 2016 & Risk Management Policy of Telecommunication and Engineering Laboratory. (12) \\
\hline Nazia chaudry, 2013 & $\begin{array}{l}\text { Assessment of biosafety practices in undergraduate \& postgraduate Students in an } \\
\text { academic institute in Islamabad.(10) }\end{array}$ \\
\hline Sadia Nasim Ahmed, 2013 & $\begin{array}{l}\text { Biosafety Intervention and Follow up Assessment in Laboratory Workers of Karachi, } \\
\text { Pakistan.(4) }\end{array}$ \\
\hline Sadia Nasim, 2010 & $\begin{array}{l}\text { Practices and Awareness Regarding Biosafety Measures Among Laboratory } \\
\text { Technicians Working in Clinical Laboratories in Karachi, Pakistan. (8) }\end{array}$ \\
\hline Shamsul A. Qasmi, 2012 & Survey of Bio risk Management in Clinical Laboratories in Karachi, Pakistan. (11) \\
\hline Adel N. Zaki, 2010 & Biosafety and biosecurity measures: management of biosafety level 3 facilities. (13) \\
\hline Christian A. Devaux, 2015 & $\begin{array}{l}\text { The hidden face of academic researches on classified highly pathogenic } \\
\text { microorganisms. (14) }\end{array}$ \\
\hline Amy C. Shurtleff, 2012 & $\begin{array}{l}\text { The Impact of Regulations, Safety Considerations and Physical Limitations on Research } \\
\text { Progress at Maximum Bio containment. (15) }\end{array}$ \\
\hline Ana Cláudia Coelho, 2011 & Biological risks and laboratory-acquired infections. (16) \\
\hline Saeed Khan, 2014 & Biosafety Practices in Different Clinical Laboratories in Karachi, Pakistan. (6) \\
\hline Hansa M Goswami, 2011 & $\begin{array}{l}\text { A study on knowledge, attitude and practice Of laboratory safety measures among } \\
\text { Paramedical staff of laboratory services. (2) }\end{array}$ \\
\hline Tin Tun, 2017 & Biomedical Laboratory: Its Safety and Risk Management.(17) \\
\hline Lyled. Feisel 2005 & The Role of the Laboratory in Undergraduate Engineering Education. (18) \\
\hline \multicolumn{1}{|c|}{ Table-l. Some studies conducted in last decade about awareness of biosafety } \\
\hline
\end{tabular}

Some literature is discussed below to highlight the results of other studies conducted for biosafety awareness.

Khan described in his work in 2012 that $60 \%$ laboratory workers confirmed that they receive formal biosafety training. $20 \%$ of the participants of research received a safety handbook. Usage of personal protective equipments were $80 \%$. however $10 \%$ labs provide eye station and emergency shower facility and $60 \%$ of the participants confirmed that fire station was not available in their lab. In the category of equipments, $80 \%$ of the participants agreed that their equipments were in working order, $60 \%$ of the participants confirmed that the PPM of their equipments were carried out on regular basis and Back up cold storage was available in only $20 \%$ of the laboratories. $40 \%$ of the participants said that the maintenance of biosafety cabinets was carried out regularly in their laboratories and only $40 \%$ were unaware regarding the certification of their biosafety cabinets. Whereas proper disinfection and decontamination of biosafety cabinets was carried out in $70 \%$ of the cases. The outcome of our survey confirms a lack of practice and implementation of good biosafety practices in the clinical laboratories. ${ }^{6}$

About Knowledge, majority knew the imperative issues related with research center security like Post Exposure Prophylaxis (96.55\%) and disposing of blood tests (93.10\%) and so on. As to state of mind towards the logical procedure, all are particularly mindful about significance of defensive gadgets (i.e. Wearing Gloves) and Biomedical waste administration. As to the training in lab, the whole investigation of Goswami 2011, subject gathering (100\%) answered "YES" in each inquiry that demonstrates the great quality work 
of the research facility. The acceptance preparing on Laboratory wellbeing is critical and propelling activity for enhancing the research facility security measures. ${ }^{2}$

It was found by Jimena 2016 that $11.2 \%$ of embryologists never wear gloves when handling semen, and $19.6 \%$ never wore them while working with follicular fluid. In addition, $30 \%$ rarely or never use protection when working with liquid nitrogen. Between 23.3\% and $47.5 \%$ believe their working conditions are not comfortable. ${ }^{7}$

By and large, $73.9 \%$ of labs $(75.9 \%$ private and $71.7 \%$ open) of Karachi, Pakistan were working without composed standard working methods and, all things considered $83.4 \%$ of labs $(85.7 \%$ private and $80.8 \%$ open) did not keep up any incident records. Strikingly, $85 \%$ of respondents did not have any preparation in biosafety. This rate is generally high in the private area, which is around $88.7 \%$, contrasted with $80.8 \%$ in people in general segment. An exploration directed by Nasim, in college of Karachi in 2010, consequences of this study affirm an absence of mindfulness with respect to great lab practices and biosafety measures among lab experts in Karachi, Pakistan, and also a requirement for sorting out essential preparing projects to build attention to great research center systems and self-hygienic standards. ${ }^{8}$

Sadia Nasim conducted research in 2012 reveled that laboratory technicians in Pakistan lack awareness of good laboratory practices and biosafety measures, and also face a lack of resources. $^{9}$

In most of the individual aspects, a significant difference in knowledge about biosafety precautions in our two groups could not be detected, described by Chaudary, 2013, however, in general awareness levels, discovered that postgraduate researchers were fundamentally better, which can be credited to their higher rate having present or past lab encounter. The examination features the need awareness sessions for all groups and for obligatory biosafety courses as ahead of schedule as conceivable in their course attempts to influence their lab to encounter safe. ${ }^{10}$

This study Qasmi, 2012 reflects the current level of Biosafety practices in clinical laboratories in Karachi, Pakistan, where major gaps were identified in all the critical areas of Biosafety and Biosecurity, including risk assessment, standard microbiological practices, primary barriers/personal protective equipment, and laboratory design/secondary barriers, and select agent protocols. The gaps included, Biosecurity is not at all defined or understood, There is no protocol/guidance for reporting laboratory acquired infections (LAls), There is no framework for registering these laboratories before commissioning, Limited or no training for Biosafety/Biosecurity is available on a continual basis, No regulatory body oversees Bio risk/Biosecurity activities or lab registration. It revealed that the degree of negligence regarding these requirements for clinical laboratories is very serious and work on an emergency basis is required to introduce, implement, and furnish Biosafety and Biosecurity guidelines. Training and literature in both English and the local Urdu language need to be developed and distributed. ${ }^{11}$

A survey published by Ahmed in 2013 in which total of 253 participants (168 males and 85 females) returned the filled questionnaire Almost all (98.8\%) laboratory workers had correct concept on use of personal protective equipment, $85 \%$ were neither practicing mouth pipetting for chemical and biological samples, nor using laboratories for eating and drinking purposes while importance of SOP/BOP and laboratory accident record maintenance was acknowledged by over 95\%. Knowledge about discarding the used syringe was present in only $68 \%$. A comparison between public and private sector laboratory workers revealed that $37 \%$ public sector workers were still discarding syringes in the dustbin as opposed to $3 \%$ from private sector. $^{4}$

Reporting of Laboratory Accidents In Pakistan multiple accidents of chemicals are 
reported most of them have been taken place in factories, filling station, road side, home areas and winning areas. ${ }^{1}$ But the dilemma is that in Pakistan, laboratory accidents reports are not apparent on any type of media and this phenomenon actually harms the input of researchers. Not a single article showed the statistics of laboratory accidents in Pakistan, this does not evade the risks of accidents but fuel the chances of negligence in laboratory safety measures.

\section{Outcomes of Reporting Timely}

The reporting of any unusual incident is a must. Specifically laboratory accidents so that a novice person in the field can prepare him/herself for upcoming vulnerabilities. And it is necessary so that authorizing bodies which are responsible for the management of biosafety among laboratories should be aware of such incident so that they can purpose and implement measures accordingly.

\section{Gaps Between the Education and Practice in Laboratories}

Though above mentioned organizations and many other institutes are spreading biosafety awareness very well across the country, but still there are gaps between the training and its implementation in the actual places. The gaps included the following: the biosafety officer is appointed in only a few laboratories, standard operating procedures are not in place in many laboratories, no training protocol is defined for staff, no immunization record is maintained, and incident reporting and recording is lacking at most laboratories. There is an adequate supply of simple personal protective equipment like gloves, goggles, masks, and lab coats. However, complex equipment, such as eyewash stations and biological safety cabinets, is not available in many laboratories. In Pakistan, biosafety performance is compromised in most of the laboratories, but better performance also is demonstrated by laboratories that are accredited and certified.

\section{SUMMARY/CONCLUSION}

Hazard prevention and control systems for specific laboratory processes must be readily shared between lab workers, their colleagues, and lab supervisors. In order for these control systems to be effective in a transferable and sustainable way, effective risk management communication tools must be present. These tools need to be adaptable and sustainable as research processes change in response to evolving scientific needs in discovery based laboratories. Responsiveness was high there were inadequacies in the zones of risk identification and disaster response. Approaches and practices were satisfactory however required change, with a frail connection existing between these two factors. The qualities of the specific establishment were observed to be the main indicator of the probability of accidents. It was reasoned that more instruction and preparing should be executed for development.

The Aga Khan University Examination Board (AKU-EB) intends to make chances to give students hands-on understanding of research facility tests which could conquer any hindrance between hypothetical ideas and its application in regular day to day existence. The research center execution depends on the possibility that science concentrates on hands-on, minds-on observational exercises, and that these exercises help students in making associations between different logical ideas and genuine practices.

Copyright(C) 15 Aug, 2018.

\section{REFERENCES}

1. de Bie P, Hui J. Current quality status of CABETs environmental laboratory and necessary steps to meet GLP requirements. Alterra/ICAMA, 2007.

2. Goswami HM, Soni ST, Patel SM, Patel MK. A study on knowledge, attitude and practice of laboratory safety measures among paramedical staff of laboratory services. Natl J Community Med. 2011;2(3):470-3.

3. Lab Safety \& Chemical Hygiene Program. Santa Barbara Santa Barbara: University of California; 2014 [cited 2017 14th december]; Available from: http:// www.ehs.ucsb.edu/labsafety/laboratory-accidents.

4. Ahmed SN, Shahid A, Mustufa MA. Biosafety intervention and follow up assessment in laboratory workers of Karachi, Pakistan. Pakistan Journal of Medical Research. 2013;52(4):106.

5. Nasim A. E3. Biotechnology and biosecurity initiatives in Pakistan: A Country Report. National Academy of Sciences; 2012 [cited 201717 December]; Available from: https://www.ncbi.nlm.nih.gov/books/ 
NBK196149/.

6. Khan S, Zehra F, Maqsood N, Zahid M, Ahmed B. Biosafety practices in different clinical laboratories in Karachi, Pakistan. Journal of Dow University of Health Sciences. 2014;8(3).

7. Jimena P, López-Lería B, Clavero A, Gonzalvo MC, Serrano M, López-Regalado ML, et al. Occupational safety in the embryology laboratory. Medicina Reproductiva y Embriología Clínica. 2016;3(2):85-91.

8. Nasim S, Shahid A, Mustufa MA, Kazmi SU, Siddiqui TR, Mohiuddin S, et al. Practices and awareness regarding biosafety measures among laboratory technicians working in clinical laboratories in Karachi, Pakistan. Applied Biosafety. 2010;15(4):172-9.

9. Nasim S, Shahid A, Mustufa MA, Arain GM, Ali G, Talreja $\mathrm{KL}$, et al. Biosafety perspective of clinical laboratory workers: a profile of Pakistan. The Journal of Infection in Developing Countries. 2012;6(08):611-9.

10. Chaudry N, Arif S, Qudsia S, Urooj S. Assessment of biosafety practices in undergraduate \& postgraduate students in an academic institute in Islamabad. Mintage Journal of Pharmaceutical and Medical Sciences. 2013:1-4.

11. Qasmi SA, Khan E, Maqbool AZ. Survey of biorisk management in clinical laboratories in Karachi, Pakistan. Applied Biosafety. 2012;17(4):198-207.
12. Shah AS, Fayaz M, Shah A, Shah S. Risk Management Policy of Telecommunication and Engineering Laboratory. International Journal of Hybrid Information Technology (IJHIT). 2016;9(4):281-90.

13. Zaki AN. Biosafety and biosecurity measures: Management of biosafety level 3 facilities. International journal of antimicrobial agents. 2010;36:S70-S4.

14. Devaux CA. The hidden face of academic researches on classified highly pathogenic microorganisms. Infection, Genetics and Evolution. 2015;29:26-34.

15. Shurtleff AC, Garza N, Lackemeyer M, Carrion Jr R, Griffiths A, Patterson J, et al. The impact of regulations, safety considerations and physical limitations on research progress at maximum biocontainment. Viruses. 2012;4(12):3932-51.

16. Coelho AC, García Díez J. Biological risks and laboratory-acquired infections: a reality that cannot be ignored in health biotechnology. Frontiers in bioengineering and biotechnology. 2015;3:56.

17. Tun T. Biomedical Laboratory: Its Safety and Risk Management. Biomedical Science Letters. 2017;23(3):155-60.

18. Feisel LD, Rosa AJ. The role of the laboratory in undergraduate engineering education. Journal of Engineering Education. 2005;94(1):121-30.

\section{AUTHORSHIP AND CONTRIBUTION DECLARATION}

\begin{tabular}{|c|l|l|}
\hline Sr. \# & \multicolumn{1}{|c|}{ Author-s Full Name } & \multicolumn{1}{|c|}{ Contribution to the paper } \\
\hline 1 & M. Sikander Ghayas Khan & Primary author \\
2 & Arif Malik & Supervisor \\
3 & Gull-e-faran & Co-author \\
4 & Madiha Fayyaz & Corresponding author \\
5 & Madiha Sikander & Super visor \\
6 & Qurrat-ul-Ain & Co-author \\
7 & Rabia Ghayas & Co-author \\
8 & Malik Muhammad Qasim & Co-author
\end{tabular}

